\title{
Índice para avaliar a eficiência da gestão da propriedade intelectual em pequenas e médias empresas
}

\section{Index to assess the efficiency of intellectual property management in small and medium-sized enterprises}

\author{
Francisco Valdivino Rocha Lima Doutor em Ciência da Propriedade Intelectual. Instituto Federal do Piauí (IFPI) - Brasil. \\ valdivino@ifpi.edu.br. \\ João Antonio Belmino dos Santos Doutor em Engenharia de Processos. Universidade Federal de Sergipe (UFS) - Brasil. \\ joaoantonio@ufs.br.
}

\section{RESUMO}

Nas pequenas e médias empresas (PMEs) proteger as inovações é fundamental para a competitividade. Entretanto, um pequeno percentual de PMEs faz uso efetivo de estratégias de gestão da propriedade intelectual, devido, entre outros fatores, à falta de ferramentas gerenciais específicas. Neste contexto, o objetivo deste artigo é desenvolver um índice para avaliar a eficiência e dar suporte às PMEs na gestão da propriedade intelectual, com base em um modelo de avaliação composto por indicadores. Para tanto, utilizouse o método Analytic Hierarchy Process (AHP), para atribuir pesos aos elementos que compõem o referido modelo. Em seguida, com base nos pesos atribuídos, desenvolveu-se a modelagem matemática do índice, bem como parâmetros para a classificação do nível de eficiência das PMEs. Os resultados apontam que o índice proposto (intitulado Índice E) foi constituído por multiatributos, distribuídos em 3 níveis (3 dimensões, 9 fatores-chave de avaliação e 42 indicadores), contemplando diversos aspectos que envolvem a dinâmica das PMEs, no tocante à gestão da propriedade intelectual.

Palavras-chave: Gestão da propriedade intelectual. Indicadores de desempenho. Índice de eficiência. Pequenas e médias empresas.

\begin{abstract}
In small and medium-size enterprises (SMEs), protecting innovations is essential for competitiveness. However, a small percentage of SMEs make effective use of intellectual property management strategies, due, among other factors, to the lack of specific management tools. In this context, the objective of this article is to develop an index to evaluate efficiency and support SMEs in the management of intellectual property based on an evaluation model composed of indicators. For this, the Analytic Hierarchy Process (AHP) method was used to assign weights to the elements that compose the model. Then, based on the assigned weights, mathematical modeling of the index was developed, as well as parameters for the classification of the level of efficiency of SMEs. The results indicate that the proposed index (entitled Index E) was made up of multi-attributes, distributed in 3 levels ( 3 dimensions, 9 key assessment factors and 42 indicators), covering several aspects that involve the dynamics of SMEs with regard to the management of intellectual property.
\end{abstract}

Keywords: Intellectual property management. Performance indicators. Efficiency index. Small and mediumsize enterprises.

Recebido em 11/07/2020. Aprovado em 03/09/2020. Avaliado pelo sistema double blind peer review. Publicado conforme normas da ABNT. http://dx.doi.org/10.22279/navus.2020.v10.p01-20.1345 


\section{INTRODUÇÃO}

A avaliação do desempenho organizacional é uma atividade essencial para o suporte no processo decisório, tanto decisões de natureza estratégica quanto táticas e operacionais, incluindo todas as perspectivas que integram as organizações, como recursos humanos, finanças, relações com o mercado, operações, inovação etc. (DEV et al., 2019; PFISTER; JACK; DARWIN, 2017). Nas empresas de grande porte ou nas pequenas e médias empresas, não é possível a melhoria da eficiência e eficácia dos processos, produtos e serviços sem uma adequada avaliação do desempenho (KAPLAN; NORTON, 2007; NEELY, 2005; SLACK et al., 2006).

Estudos apontam que, no âmbito das pequenas e médias empresas (PMEs), a avaliação de desempenho tem um papel fundamental no apoio gerencial, e diversos trabalhos propõem ferramentas com o objetivo de contribuir com a gestão dessas empresas (MAHMOOD et al., 2018; PARK, 2014; SHI et al., 2013). Entretanto, pesquisas relacionadas à avaliação de desempenho da gestão da propriedade intelectual (PI) em PMEs são incipientes. Em uma revisão sistemática da literatura acerca do tema, Lima e Santos (2018) identificaram apenas dois artigos com esse propósito (ENJOLRAS et al., 2014; PETIT et al., 2011).

Para mensurar a eficiência da gestão da propriedade intelectual nas PMEs, assim como em qualquer outra área estratégica de uma organização, o meio mais utilizado são os indicadores, os quais devem estar alinhados com as estratégias do negócio e fornecer informações essenciais para a tomada de decisão (BOGETOFT, 2013; COHEN et al., 2012). Os indicadores de desempenho auxiliam na definição de metas e a comparação dessas metas com informações do mercado, para identificar em quais aspectos a empresa está alcançando melhores resultados, bem como aqueles nos quais precisa melhorar (LO-IACONO-FERREIRA; CAPUZ-RIZO; TORREGROSA-LÓPEZ, 2018; SAUNILA, 2016).

Um dos principais equívocos quando se trata de indicadores de desempenho é abordá-los como sinônimos de índices, pois a diferença entre esses dois termos é acentuada. Enquanto o indicador "evidencia" e "destaca" um dado fenômeno; o índice "sinaliza", por meio de uma grandeza (medida-síntese), tanto um elo de proximidade com o representado, quanto o aumento ou redução de uma quantidade em relação a um padrão. Os índices, portanto, têm a função de agregar um conjunto de indicadores, de uma mesma dimensão ou diferentes dimensões de um fenômeno (BELLEN, 2005; JANNUZZI, 2005; JOUNG et al., 2013; OCDE, 2003).

O desenvolvimento de índices é uma técnica relevante para agregar e sintetizar informações que integram um número expressivo de indicadores, que, de forma isolada, são difíceis de analisar. Na construção de índices, sobretudo de desempenho organizacional, utilizam-se metodologias para atribuir pesos relativos a cada dimensão que compõe o sistema de indicadores e para cada indicador individualmente, a fim de, a partir desses pesos, definirem-se escalas de variáveis em uma medida-síntese única, que se transforma em medidas de base e referência (BELLEN, 2005; JANNUZZI, 2005; MA et al., 2011; WANG; HWANG, 2011).

Ante o exposto, o objetivo deste artigo foi desenvolver, a partir de um conjunto de indicadores validados, um índice gerencial para mensurar a eficiência da gestão da propriedade intelectual em pequenas e médias empresas (PMEs). Diversas variáveis que envolvem a dinâmica das PMEs, no tocante à gestão da propriedade intelectual, foram sintetizadas no índice proposto (intitulado Índice E). A escolha das PMEs como objeto de estudo no presente trabalho justifica-se porque essas empresas são indutoras do desenvolvimento econômico em diversos países, por meio da geração de um número significativo de postos de trabalho e por uma expressiva geração de receitas tributárias (EJDYS, 2014), necessitando, portanto, de ferramentas gerenciais em áreas estratégicas como a gestão da propriedade intelectual, a fim de se tornarem cada vez mais competitivas.

Para atingir o objetivo proposto, este trabalho está estruturado da seguinte forma: a introdução, que destaca a importância da avaliação do desempenho no contexto organizacional; na seção 2 são descritos os procedimentos metodológicos; os resultados e as discussões encontram-se na seção 3; e na seção 4 são apresentadas as considerações finais. 


\section{GESTÃO DA PROPRIEDADE INTELECTUAL EM PEQUENAS E MÉDIAS EMPRESAS}

O principal recurso de uma organização no atual cenário econômico é o capital intelectual, formado pelo capital humano (conhecimento, habilidades e competências das pessoas) e pelos ativos intelectuais (que abrangem os conhecimentos que foram codificados de alguma forma). No âmbito dos ativos intelectuais há um subconjunto de conhecimentos, resultante do processo inovativo, chamado de propriedade intelectual (CANDELIN-PALMQVIST; SANDBERG; MYLLY, 2012; HARRISON; SULLIVAN, 2011). A gestão da propriedade intelectual é um conjunto de ações estratégicas e operacionais, com ênfase na identificação, aquisição, proteção e apropriação dos direitos relativos a produtos e processos inovadores (AGOSTINI; NOSELLA; SORANZO, 2015; REITZIG, 2007).

No contexto das pequenas e médias empresas, assim como nas organizações de grande porte, a gestão da propriedade intelectual deve estar integrada e alinhada às diretrizes estratégicas do negócio (missão, visão, valores, objetivos e metas) e com os planos táticos e operacionais das diversas áreas funcionais (marketing, finanças, operações e recursos humanos) que a integram e que são responsáveis pela implementação das estratégias de toda a organização, ajustando-se à medida que o negócio avance nos diferentes estágios de seu desenvolvimento (FREEL; ROBSON, 2017; MOL; MASUREL, 2011).

As pequenas e médias empresas podem aplicar diversos métodos para proteger seus ativos de propriedade intelectual, visto que diferentes mecanismos podem ser utilizados de forma combinada quando as inovações tecnológicas são compostas por componentes possíveis de serem protegidos separadamente (HALL; SENA, 2017; LANDRY; AMARA; SAIHI, 2009). Achados na literatura apontam duas categorias de métodos de proteção: (1) mecanismo de proteção formal (baseado na legislação de cada país e em acordos e tratados internacionais), que inclui patentes; desenhos industriais; registro de marcas, direitos autorais etc.; e (2) mecanismos de proteção informal (extralegal), que abrange segredo comercial, manutenção da qualidade do produto, complexidade do design, uso de exclusividade, retenção de pessoal qualificado etc. (KELLI et al., 2010; MOL; MASUREL, 2011; THOMÄ, 2013).

A escolha do método de proteção e apropriação está condicionada a fatores como o grau e o tipo de inovação, o modelo organizacional e o ambiente geral do mercado. Em outras palavras, depende do contexto e do processo organizacional, dos objetivos corporativos e das características da tecnologia utilizadas pelas empresas (HALL; SENA, 2017; WILLOUGHBY, 2013). Por exemplo, uma parte expressiva do conhecimento operacional em pequenas empresas tende a ser tácito, funcionando, portanto, como método de apropriação efetiva. Além disso, as patentes podem não estar disponíveis para grande número de pequenas empresas, precisamente porque sua base de conhecimento tácito não pode ser reduzida a informações codificadas (THOMÄ, 2013).

A gestão da propriedade intelectual permite às PMEs o fortalecimento das competências organizacionais com foco na proteção e apropriação dos resultados do esforço inovativo (EPPINGER; VLADOVA, 2013), por meio da criação de barreira para a entrada de concorrentes em determinado nicho de mercado; ou pela viabilização de acesso permanente a tecnologias estratégicas. Para isso, é fundamental o direcionamento de esforços para os aspectos comerciais, colaborativos, financeiros, humanos e administrativos, relativos aos bens de propriedade intelectual (LAWSON; SAMSON; RODEN, 2012).

Em relação aos aspectos de natureza colaborativa e comercial, Wang (2016) enfatiza que a gestão da propriedade intelectual deve ter como parâmetro as demandas impostas pelo ambiente externo. Neste sentido, considerando as características das PMEs, que contempla a limitação de recursos para investimentos, é imprescindível a adoção de diversas formas de alianças, como colaboração em P\&D, terceirização, concessão de licenças ou cessão, parceria público-privada etc., inclusive por meio de cooperação em rede com empresas maiores, universidades e centros de pesquisas (KÖHLER, 2011; HU; TSAI, 2006). Essas alianças proporcionam obtenção de recursos humanos, tecnológicos e financeiros, assegurando a apropriabilidade, por meio do acesso a mercados, parceiros e conhecimentos estratégicos, reduzindo os custos e os riscos da inovação (REHMAN et al., 2016).

No tocante aos aspectos relativos aos recursos humanos, Thomä e Zimmermann (2013) destacam a retenção de pessoal qualificado como um dos mais importantes mecanismos de proteção da inovação para as 
PMEs. Essa estratégia de proteção consiste, sobretudo, em práticas específicas de gestão de pessoas, implementadas pelas empresas, com o objetivo de aumentar o comprometimento dos funcionários, com foco na conscientização e estímulo à inovação. Ações dessa natureza podem incluir a criação de oportunidades de aprendizado e planos de remuneração adequados, valorização e reconhecimento das contribuições dos empregados e a provisão de oportunidades de carreira, a partir do mapeamento das competências e de uma adequada avaliação de desempenho do corpo funcional (DELERUE; LEJEUNE, 2011; KELLI et al., 2010; DODGE et al., 2017; TALVELA et al., 2016).

Para Schauten e Stegink (2013), os aspectos de natureza financeira relacionados aos bens de propriedade intelectual devem ser tratados de forma estratégica pelas PMEs. Segundo eles, esses bens, quando avaliados, valorados e gerenciados de forma eficiente, ampliam de forma expressiva o valor do patrimônio líquido das organizações. Além disso, podem ser utilizados para favorecer a obtenção de financiamentos, bem como informar a viabilidade econômica dessas empresas aos investidores (CHEN, 2011).

Por fim, ressalta-se que nas pequenas e médias empresas, as ações operacionais e administrativas voltadas para a construção e manutenção de um portfólio de ativos protegidos devem ser implementadas de forma contínua, inclusive com a adoção de indicadores para mensurar o desempenho nessas atividades (LAWSON; SAMSON; RODEN, 2012; SEO et al., 2015; TIETZE; GRANSTRAND; HERSTATT, 2006). Essas aç̃̃es devem contemplar, além da gestão de contratos de transferência de tecnologia e licenciamentos, a auditoria de todas as ações relativas à proteção de ativos de propriedade intelectual, permitindo a minimização dos riscos de prejuízo devido às práticas ilegais dos concorrentes, redução de custos, otimização de esforços, aumento da eficiência e eficácia, bem como a maximização da produtividade no processo inovativo (ACKERMAN, 2018).

\section{PROCEDIMENTOS METODOLÓGICOS}

O índice para avaliar a eficiência da gestão da propriedade intelectual em PMEs (Índice $E$ ) foi desenvolvido com base em um modelo conceitual composto por dimensões, fatores-chave de avaliação e indicadores, elaborado por Lima (2019), por meio de uma revisão sistemática da literatura e validado mediante a aplicação do método Fuzzy Delphi. O desenvolvimento do Índice E contemplou duas etapas, conforme apresentado na Figura 1.

Figura 1 - Etapas da pesquisa

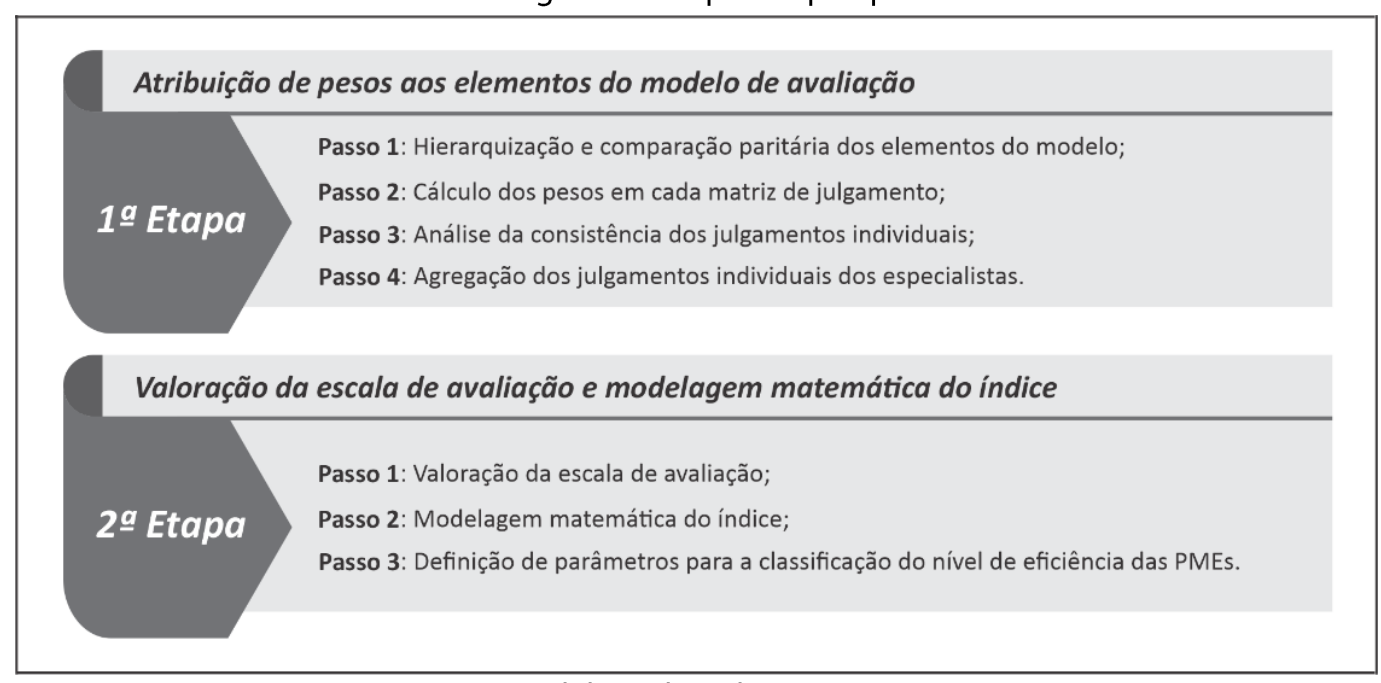

Fonte: Elaborado pelos autores (2020). 


\section{1a Etapa: Atribuição de pesos aos elementos do modelo de avaliação}

Aplicou-se o método Analytic Hierarchy Process (AHP) para atribuir pesos aos elementos que integram o modelo validado (dimensões, fatores-chave de avaliação e indicadores). Este método foi adotado no presente trabalho por se tratar de uma ferramenta multicritério de tomada de decisão que auxilia no julgamento de problema, decompondo-o em uma estrutura hierárquica de vários níveis, incluindo objetivos, critérios, subcritérios e alternativas (SAATY, 1990); e por ser apontado em diversas pesquisas como o método mais indicado para a priorização de múltiplos indicadores (MARDANI et al., 2015; SEKHAR; PATWARDHAN; VYAS, 2015). Para este estudo, a aplicação do método AHP foi dividida em 4 passos:

\section{Passo 1: Hierarquização e comparação paritária dos elementos do modelo}

Inicialmente, construiu-se a estrutura hierárquica do Índice $E$ (Figura 2). Em seguida, realizou-se a comparação paritária por meio de um questionário estruturado. Foram convidados 15 profissionais brasileiros com formação ou experiência na área de propriedade intelectual; ou com experiência em consultoria com foco em inovação para PMEs, dos quais 14 concluíram as comparações.

Os especialistas realizaram a comparação com base na importância relativa de cada elemento em seu respectivo nível, criando-se, dessa forma, matrizes recíprocas de decisão. Para isso, utilizou-se a escala fundamental, desenvolvida por Saaty (1990), na qual emprega-se valores de 1 a 9, em que 1 consiste em igual preferência e 9 representa elevado grau de preferência (Quadro 1).

Quadro 1 - Escala fundamental de Saaty

\begin{tabular}{|c|c|c|}
\hline $\begin{array}{l}\text { Intensidade de } \\
\text { Importância }\end{array}$ & Definição & Explicação \\
\hline 1 & Igual importância & $\begin{array}{l}\text { As contribuições de dois elementos são igualmente } \\
\text { importantes. }\end{array}$ \\
\hline 3 & $\begin{array}{l}\text { Importância fraca de uma } \\
\text { sobre a outra }\end{array}$ & $\begin{array}{l}\text { Experiência e julgamento privilegiam discretamente um } \\
\text { elemento em detrimento de outro. }\end{array}$ \\
\hline 5 & $\begin{array}{c}\text { Importância grande ou } \\
\text { essencial }\end{array}$ & $\begin{array}{l}\text { Experiência e julgamento privilegiam significativamente } \\
\text { um elemento em detrimento de outro. }\end{array}$ \\
\hline 7 & Importância muito grande & $\begin{array}{l}\text { Um elemento é fortemente privilegiado em detrimento } \\
\text { de outro, podendo ser demonstrado na prática. }\end{array}$ \\
\hline 9 & Importância absoluta & $\begin{array}{l}\text { A evidência privilegia um elemento em relação ao outro, } \\
\text { de forma extremamente contundente. }\end{array}$ \\
\hline $2,4,6$ e 8 & $\begin{array}{l}\text { Valores em posição } \\
\text { intermediária }\end{array}$ & $\begin{array}{l}\text { Aplicado em caso de dúvida entre dois valores de } \\
\text { intensidade. }\end{array}$ \\
\hline
\end{tabular}

Fonte: Adaptado de Saaty (1990).

Segundo Saaty (1990), na condução de uma comparação paritária, para n elementos que estão sendo avaliados, serão necessárias $\rho$ comparações, conforme Equação 1.

$$
\boldsymbol{\rho}=n \frac{(n-1)}{2}
$$

Ainda de acordo com Saaty (1990), os resultados de comparação de nelementos devem ser colocados na parte triangular superior da matriz de comparação, enquanto a parte triangular inferior serve como o valor reverso do valor relativo da parte triangular superior. Feito isso, obtêm-se uma matriz de comparação de pares A, conforme mostrado na Equação 2. 


$$
A=\left[\begin{array}{cccc}
1 & a_{12} & \cdots & a_{1 n} \\
1 / a_{12} & 1 & \cdots & a_{2 n} \\
\vdots & \vdots & \ddots & \vdots \\
1 / a_{1 n} & 1 / a_{2 n} \cdots & \cdots & 1
\end{array}\right]
$$

na qual $a_{i j}$ representa a importância relativa do elemento i em uma avaliação comparativa com o elemento $j$.

Passo 2: Cálculo dos pesos em cada matriz de julgamento

Após a realização dos julgamentos paritários, foi calculado o autovetor das matrizes, por meio da média geométrica de cada linha dessas matrizes. Em seguida, fez-se a normalização da matriz de julgamento, através da divisão do autovetor de cada linha pela soma da coluna do autovetor, obtendo-se os pesos (HUANG; LAl; LIN, 2011; WANG; HWANG, 2011), como apresentado na Equação 3.

$$
W_{i}=\frac{\sqrt[n]{\prod_{j=1}^{n} a_{i j}}}{\sum_{i=1}^{n} \sqrt[n]{\prod j=1^{n} a_{i j}}} i, j=1,2, \ldots, n .
$$

Passo 3: Análise da consistência dos julgamentos individuais

Para Saaty (1990), os julgamentos em uma matriz paritária podem ser incoerentes. Por esse motivo, ele criou dois índices: Índice de coerência (IC) e Razão de Consistência (RC). Para obtê-los, inicialmente calculou-se o $\lambda$ max, por meio da multiplicação da matriz de comparação $A$ pelo autovetor $W_{i}$, para obter um novo autovetor, $W_{i}^{\prime}$, como apresentado na Equação 4; em seguida, cada valor de autovetor de $W_{i}^{\prime}$ foi dividido por cada valor do autovetor original $W_{\mathrm{i}}$. Dessa forma, usou-se todos os valores obtidos para calcular uma média aritmética, derivando $\lambda \max$ (WANG; HWANG, 2011), como mostrado na Equação 5.

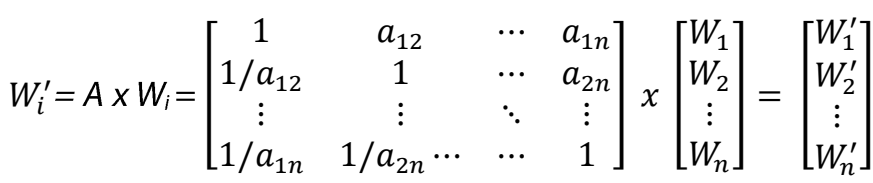

$$
\begin{aligned}
& \lambda_{\max }=\frac{1}{n}\left(\sum_{i=0}^{n} \frac{w_{i}^{\prime}}{w_{i}}\right)
\end{aligned}
$$

Com base no $\lambda$ max foi possível calcular Índice de coerência (IC) e Razão de Consistência (RC), representados, respectivamente, pelas Equação 6 e 7:

a) Índice de Coerência (IC):

$$
I C=\frac{\lambda_{\max }-n}{n-1}
$$

em que $n$ é a ordem da matriz.

b) Razão de Coerência (RC):

$$
R C=\frac{I C}{I R}
$$


em que IC é Índice Randômico, estabelecido por Saaty (1990), conforme ordem das matrizes, como fornecido pela Tabela 1.

Tabela 1 - Índice de consistência randômico

\begin{tabular}{c|c|c|c|c|c|c|c|c|c|c}
\hline Ordem da matriz & $\mathbf{1}$ & $\mathbf{2}$ & $\mathbf{3}$ & $\mathbf{4}$ & $\mathbf{5}$ & $\mathbf{6}$ & $\mathbf{7}$ & $\mathbf{8}$ & $\mathbf{9}$ & $\mathbf{1 0}$ \\
\hline $\mathbf{I R}$ & 0,00 & 0,00 & 0,58 & 0,90 & 1,12 & 1,24 & 1,31 & 1,41 & 1,45 & 1,49 \\
\hline \multicolumn{10}{c}{ Fonte: Saaty $(1990)}$.
\end{tabular}

Saaty (1990) assevera que, quanto menor o valor de RC, mais consistentes são os julgamentos, portanto, para $\mathrm{RC}=0$, tem-se consistência plena. De um modo geral, para $\mathrm{RC}<10 \%$ os julgamentos são coerentes.

Ressalta-se que julgamentos inconsistentes foram repetidos e/ou excluídos do processo de agregação, a fim de se obter a coerência exigida pelo método AHP. Nesta pesquisa, foram adotados os seguintes critérios para exclusão e/ou solicitação de reconsideração de uma comparação paritária:

a) Solicitação de reavaliação: matrizes de comparação com RC maior que $10 \%$ e menor ou igual a $50 \%$;

b) Exclusão do processo de agregação: matrizes de comparação avaliadas inicialmente com RC maior que $50 \%$ ou reavaliadas com $\mathrm{RC}$ maior que $10 \%$.

\section{Passo 4: Agregação dos julgamentos individuais dos especialistas}

Os julgamentos dos 14 especialistas foram agrupados para a consolidação da análise. Para isso, optouse pela técnica AIJ (Aggregating Individual Judgments). Por meio dessa técnica, a agregação é feita pelo cálculo da média geométrica de cada posição das matrizes de julgamento, resultando em uma nova matriz global (FORMAN; PENIWATI, 1998).

\section{2a Etapa: Valoração da escala de avaliação e modelagem matemática do índice}

A finalidade da presente pesquisa foi desenvolver um índice geral para avaliar o desempenho das PMEs contem plando os 9 fatores-chave propostos no modelo de Lima (2019). Por esse motivo, e considerando que na etapa anterior os pesos foram atribuídos aos fatores-chave no âmbito de suas respectivas dimensões, nesta etapa esses pesos foram normalizados com base nos pesos atribuídos a essas dimensões. Já os pesos atribuídos aos indicadores foram mantidos sem normalização, a fim de nivelar o índice geral ao índice de cada fator-chave de avaliação. Esta etapa foi conduzida em 3 passos:

Passo 1: Valoração da escala de avaliação

Neste passo, a escala de avaliação de cada indicador recebeu uma pontuação relativa, em um procedimento semelhante ao adotado por Marras (2012), conforme os critérios a seguir:

a) As escalas de avaliação dos indicadores propostos no modelo de Lima (2019) variam de 1 a 5 níveis. Assim, os indicadores foram organizados de acordo com seu respectivo fator-chave, em uma tabela, na qual foi arbitrado o valor 0,1 (zero vírgula um) para o somatório da coluna relativa ao primeiro nível 1 (N1) da escala de avaliação.

b) Experiências laboratoriais de construção de tabelas têm evidenciado que a otimização deste instrumento de medição como sistema se consegue ao aplicar uma razão $\left(r^{\prime}\right)$ entre 8 e 12 nos valores dos somatórios do primeiro e último nível. Para este estudo, adotou-se $r^{\prime}=10$. Dessa forma, o somatório da coluna da tabela relativo ao último nível equivale a 1,0 (um). Assim, o valor do índice de cada fator-chave de avaliação, bem como do índice geral, foi limitado ao intervalo de 0,1 a 1,0; sendo que estes valores representam o mais baixo e o mais alto desempenho possível para o índice, respectivamente.

c) Para cada indicador, o valor do primeiro e do último nível da escala de avaliação foi calculado multiplicando-se o somatório do nível correspondente (primeiro ou último) pelo peso atribuído ao referido indicador por meio do método AHP. 
d) Os valores intermediários da escala de avaliação de cada indicador foram calculados por meio de uma interpolação geométrica, multiplicando-se o valor do nível 1 (N1) por uma constante ( $r$ "), e assim sucessivamente, até que todos os níveis da escala estivessem com os valores intermediários atribuídos. A constante de multiplicação r" foi calculada pela Equação 8:

$$
r^{\prime \prime}=\sqrt[n-1]{\frac{b}{a}}
$$

em que:

$\mathrm{a}=$ valor do primeiro nível da escala de avaliação de cada indicador;

$\mathrm{b}=$ valor do último nível da escala de avaliação de cada indicador;

$\mathrm{n}=$ número de níveis da avaliação da escala;

$r^{\prime \prime}=$ constante multiplicador.

Passo 2: Modelagem matemática do índice

Com base na escala de avaliação valorada, foi desenvolvida a modelagem matemática do Índice de Eficiência da Gestão da Propriedade Intelectual em Pequenas e Médias Empresas (Índice E), que representa uma função do desempenho das PMEs nos nove fatores-chave de avaliação.

\section{Passo 3: Definição de parâmetros para a classificação do nível de eficiência das PMEs}

No passo 3, definiram-se intervalos para a classificação do nível de eficiência das PMEs na gestão da propriedade intelectual, por meio de cinco graus (I, II, III, IV e V), às quais índice proposto poderá ser associado. Os graus foram estabelecidos de forma linear no intervalo de 0,1 a 1,0 (valores que representam o mais baixo e o mais alto desempenho possível para os índices).

\section{RESULTADOS E DISCUSSÃO}

O modelo de avaliação de desempenho da gestão da propriedade intelectual em PMEs, desenvolvido por Lima (2019), a partir do qual o índice proposto no presente trabalho foi desenvolvido, é composto por três dimensões: (i) Capital humano; (ii) Contexto estratégico; e (iii) Recursos e processos. O Quadro 2 apresenta o escopo e o comparativo de referências teóricas dos fatores-chave de avaliação que integram essas dimensões.

Quadro 2-Dimensões e fatores-chave do modelo de avaliação proposto

\begin{tabular}{|c|c|c|c|}
\hline Dim. & $\begin{array}{c}\text { Fator-chave de } \\
\text { avaliação }\end{array}$ & Escopo & Referências \\
\hline \multirow{3}{*}{ 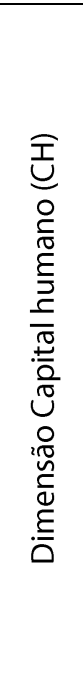 } & $\begin{array}{l}\text { Cultura e } \\
\text { aprendizagem } \\
\text { (FCA) }\end{array}$ & $\begin{array}{l}\text { Envolve os esforços para promover, pautados nas } \\
\text { diretrizes estratégicas, um ambiente } \\
\text { organizacional focado na proteção e apropriação } \\
\text { dos bens de propriedade intelectual, por meio do } \\
\text { treinamento e desenvolvimento do corpo } \\
\text { funcional. }\end{array}$ & $\begin{array}{l}\text { Dodge et al. (2017); Talvela } \\
\text { et al. (2016); Thomä e } \\
\text { Zimmermann (2013). }\end{array}$ \\
\hline & $\begin{array}{l}\text { Recompensa e } \\
\text { retenção (FRR) }\end{array}$ & $\begin{array}{l}\text { Consiste na implementação de práticas } \\
\text { específicas da gestão de recursos humanos, a fim } \\
\text { de aumentar o comprometimento do corpo } \\
\text { funcional, contemplando a criação de } \\
\text { oportunidades de aprendizado, a formatação de } \\
\text { planos de remuneração atrativos etc. }\end{array}$ & $\begin{array}{l}\text { Dodge et al., (2017); Talvela } \\
\text { et al., (2016); Thomä e } \\
\text { Zimmermann (2013). }\end{array}$ \\
\hline & $\begin{array}{l}\text { Monitoramento } \\
\text { de pessoal (FMP) }\end{array}$ & $\begin{array}{l}\text { Definição de metas para os funcionários no } \\
\text { tocante às ações de PI, bem como um } \\
\text { estabelecimento de um protocolo de conduta }\end{array}$ & $\begin{array}{l}\text { Delerue e Lejeune (2011); } \\
\text { Kelli et al. (2010); Thomä e } \\
\text { Zimmermann (2013). }\end{array}$ \\
\hline
\end{tabular}




\begin{tabular}{|c|c|c|c|}
\hline & & $\begin{array}{l}\text { acerca do sigilo de informações, a fim de } \\
\text { acompanhar a contribuição individual deles no } \\
\text { alcance dos objetivos organizacionais relativos à } \\
\text { PI. }\end{array}$ & \\
\hline \multirow{3}{*}{ 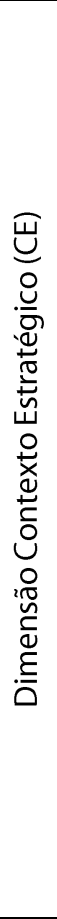 } & $\begin{array}{c}\text { Inteligência } \\
\text { tecnológica (FIT) }\end{array}$ & $\begin{array}{l}\text { Contempla a utilização de informações do } \\
\text { mercado para orientar a tomada de decisão } \\
\text { relativa à gestão da propriedade intelectual nas } \\
\text { PMEs, a fim de criar vantagem competitiva } \\
\text { sustentável, por meio do aproveitamento das } \\
\text { oportunidades a partir da aplicação eficiente dos } \\
\text { recursos disponíveis. }\end{array}$ & $\begin{array}{l}\text { Clarke e turner (2003); Lee et } \\
\text { al., (2014); Littmann-hilmer e } \\
\text { Kuckartz (2009); Regazzoni, } \\
\text { Rizzi e Nani (2011); Weenen } \\
\text { et al. (2013) }\end{array}$ \\
\hline & $\begin{array}{l}\text { Articulação } \\
\text { Externa (FAE) }\end{array}$ & $\begin{array}{l}\text { Consiste no planejamento e implementação de } \\
\text { ações de natureza estratégica, relativas à gestão } \\
\text { da propriedade intelectual, visando à } \\
\text { internalização e à externalização de } \\
\text { conhecimentos e tecnologias, por meio da } \\
\text { celebração de parcerias e alianças, com foco em } \\
\text { P\&D e na apropriação das inovações } \\
\text { desenvolvidas pela empresa. }\end{array}$ & $\begin{array}{l}\text { Agostini e Nosella (2017); } \\
\text { Belingheri e Leone (2017); } \\
\text { Hu e Tsai (2006); Van } \\
\text { Rijnsoever, Kempkes e } \\
\text { Chappin (2017); Wang } \\
\text { (2016); Zubielqui, Jones e } \\
\text { Statsenko (2016). }\end{array}$ \\
\hline & $\begin{array}{c}\text { Alinhamento } \\
\text { gerencial (FAG) }\end{array}$ & $\begin{array}{l}\text { Abrange ações de gestão da propriedade } \\
\text { intelectual integradas com a estratégia global da } \\
\text { empresa e que devem se ajustar à medida que o } \\
\text { negócio se mova através de diferentes estágios } \\
\text { de seu desenvolvimento. }\end{array}$ & $\begin{array}{l}\text { Brooking (2010); Maldonado- } \\
\text { Guzmán et al., (2016); Tietze, } \\
\text { Granstrand e Herstatt (2006). }\end{array}$ \\
\hline \multirow{3}{*}{ 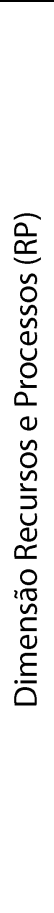 } & $\begin{array}{l}\text { Mobilização de } \\
\text { recursos (FMR) }\end{array}$ & $\begin{array}{l}\text { Consiste na prospecção, obtenção e alocação de } \\
\text { recursos (financeiros, tecnológicos e materiais) } \\
\text { necessários para dar suporte aos processos } \\
\text { operacionais de proteção e apropriação dos } \\
\text { ativos intelectuais. }\end{array}$ & $\begin{array}{l}\text { Eppinger e Vladova (2013); } \\
\text { Lawson, Samson e Roden } \\
\text { (2012); Seo et al. (2015); } \\
\text { Tietze, Granstrand e Herstatt } \\
\text { (2006). }\end{array}$ \\
\hline & $\begin{array}{l}\text { Processo de } \\
\text { proteção dos } \\
\text { ativos de PI (FPP) }\end{array}$ & $\begin{array}{l}\text { Envolve o planejamento, a organização e a } \\
\text { execução de ações vinculadas a produtos e } \\
\text { processos inovadores, por meio de instrumentos } \\
\text { formais (caracterizado por possuir base jurídica) e } \\
\text { mecanismos de proteção informais (extralegais), } \\
\text { bem como o monitoramento sistemático dos } \\
\text { direitos dos ativos protegidos. }\end{array}$ & $\begin{array}{l}\text { Delerue e Lejeune (2011); } \\
\text { Kelli et al., (2010); Landry, } \\
\text { Amara e Saihi (2009); Mol e } \\
\text { Masurel (2011); Reitzig } \\
\text { (2007); Thomä (2013); } \\
\text { Ackerman (2018). }\end{array}$ \\
\hline & $\begin{array}{l}\text { Processo de } \\
\text { apropriação da PI } \\
\text { (FPA) }\end{array}$ & $\begin{array}{l}\text { Consiste na captura de valor a partir da inovação, } \\
\text { por meio da monetização dos direitos de } \\
\text { propriedade intelectual, contemplando } \\
\text { estratégias como uso exclusivo de inovações } \\
\text { tecnológicas, concessão de licenças ou cessão, } \\
\text { cooperação público-privada, litigação, uso da PI } \\
\text { como garantia, securitização da propriedade } \\
\text { intelectual etc. }\end{array}$ & $\begin{array}{l}\text { Freel e Robson (2017); } \\
\text { Holgersson (2013); Kitching } \\
\text { e Blackburn (1998); Köhler } \\
\text { (2011); Leiponen e Byma } \\
\text { (2009); Rehman et al. (2016); } \\
\text { Shang, Qiu e Wen (2017). }\end{array}$ \\
\hline
\end{tabular}

Fonte: Elaborado pelos autores, com base em Lima (2019).

Cada um dos fatores-chave de avaliação descritos no Quadro 2 é formado por um conjunto de indicadores. Assim, o índice para avaliar a eficiência da gestão da propriedade intelectual em pequenas e médias empresas (Índice E), proposto neste trabalho, foi constituído por multiatributos, distribuídos em 3 níveis hierárquicos (3 dimensões, 9 fatores-chave de avaliação e 42 indicadores), conforme apresentado na Figura 2. 
Figura 2 - Estrutura hierárquica do Índice E

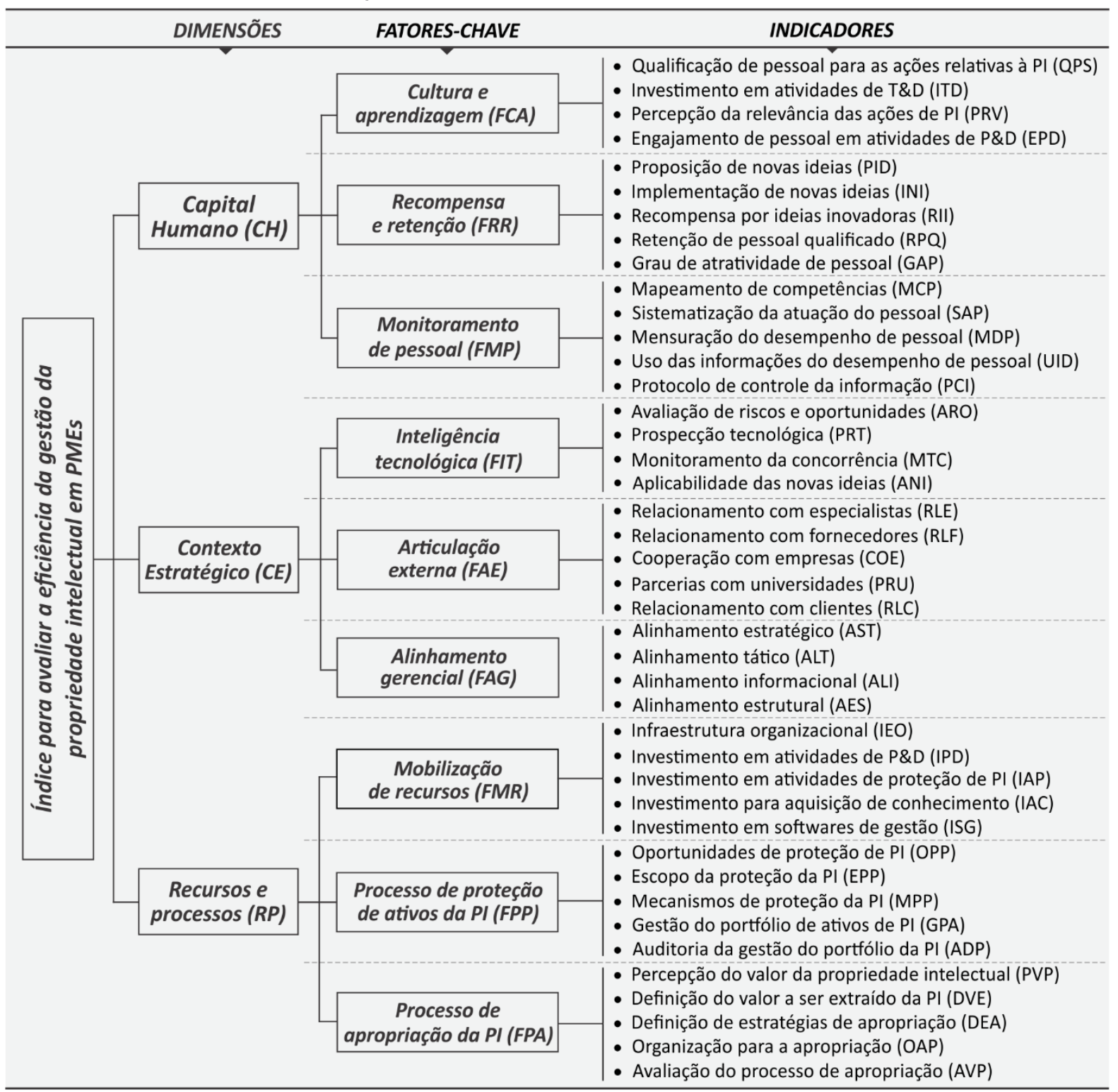

Fonte: Elaborado pelos autores, com base em Lima (2019).

Os indicadores listados na Figura 2 têm natureza quantitativa, calculados por fórmulas específicas, cujos valores poderão variar de 0,0 a 1,0, ordenados em uma escala de cinco níveis; ou natureza qualitativa, ordenados em três ou quatro níveis. As fórmulas dos indicadores quantitativos e as escalas ordinais dos indicadores qualitativos são apresentados no modelo proposto por Lima (2019).

Como descrito nos procedimentos metodológicos, para definir o valor dos níveis da escala de avaliação de cada indicador, atribuíram-se pesos para todos os atributos que integram o índice proposto, por meio do método AHP. Dessa forma, a Tabela 2 apresenta o resultado da comparação paritária das três dimensões que dão sustentação ao índice.

Tabela 2 - Matriz 1 (Comparação paritária das dimensões do Índice $E$ )

\begin{tabular}{r|c|c|c|c}
\hline \multicolumn{1}{c|}{ Dimensão } & CH & CE & RP & Peso \\
\hline Capital Humano (CH) & 1,000 & 1,695 & 1,325 & 0,428 \\
\hline Contexto Estratégico (CE) & 0,590 & 1,000 & 1,350 & 0,303 \\
\hline Recursos e Processos (RP) & 0,755 & 0,741 & 1,000 & 0,269 \\
\hline \multicolumn{1}{c|}{$\boldsymbol{\lambda} \max =3,033$} & I.C. $=0,017$ & R.C. $=0,029$ \\
\hline
\end{tabular}

Fonte: Elaborado pelos autores (2019). 
Os dados apresentados na Tabela 2, com destaque para a dimensão Capital humano (Peso $=0,428$ ), estão alinhados com o posicionamento de Delerue e Lejeune (2011) e de Thomä e Zimmermann (2013), segundo o quais, no âmbito empresarial, as pessoas são mais relevantes que os processos de estratégia e operações, visto que são elas (as pessoas) que analisam as demandas do mercado, formulam estratégias a partir dessa análise e as implementam, criando produtos, processos e serviços inovadores.

Após a comparação das dimensões que integram o Índice $E$, realizou-se a comparação dos fatoreschave de avaliação no âmbito da sua respectiva dimensão. Dessa forma, na Tabela 3 são apresentadas três matrizes de julgamento.

Tabela 3 - Matriz 1 (Comparação paritária das dimensões do Índice $E$ )

\begin{tabular}{|c|c|c|c|c|c|}
\hline \multirow{5}{*}{$\begin{array}{c}\text { Matriz } 2 \\
\text { (Capital } \\
\text { Humano) }\end{array}$} & Fatores-chave & FCA & FRR & FMP & Peso \\
\hline & Cultura e aprendizagem (FCA) & 1,000 & 1,022 & 1,794 & 0,401 \\
\hline & Recompensa e retenção (FRR) & 0,978 & 1,000 & 1,228 & 0,348 \\
\hline & Monitoramento de pessoal (FMP) & 0,557 & 0,815 & 1,000 & 0,252 \\
\hline & $\lambda \max =3,014$ & I.C. = & 0,007 & R.C. = & 0,012 \\
\hline \multirow{5}{*}{$\begin{array}{c}\text { Matriz } 3 \\
\text { (Contexto } \\
\text { Estratégico) }\end{array}$} & Fatores-chave & FIT & FAE & FAG & Peso \\
\hline & Inteligência tecnológica (FIT) & 1,000 & 1,609 & 1,368 & 0,423 \\
\hline & Articulação Externa (FAE) & 0,622 & 1,000 & 1,708 & 0,332 \\
\hline & Alinhamento Gerencial (FAG) & 0,731 & 0,586 & 1,000 & 0,245 \\
\hline & $\lambda \max =3,054$ & I.C. = & 0,027 & R.C. $=$ & 0,047 \\
\hline \multirow{5}{*}{$\begin{array}{c}\text { Matriz } 4 \\
\text { (Recursos e } \\
\text { Processos) }\end{array}$} & Fatores-chave & FMR & FPP & FPA & Peso \\
\hline & Mobilização de recursos (FMR) & 1,000 & 0,592 & 0,557 & 0,217 \\
\hline & Processo de proteção dos ativos de PI (FPP) & 1,689 & 1,000 & 0,445 & 0,285 \\
\hline & Processo de apropriação da PI (FPA) & 1,794 & 2,245 & 1,000 & 0,498 \\
\hline & $\lambda \max =3,063$ & I.C. $=$ & 0,031 & R.C. = & 0,054 \\
\hline
\end{tabular}

Fonte: Elaborado pelos autores (2019).

Os dados da Tabela 3 evidenciam que Cultura e aprendizagem (peso $=0,401$ ) é o fator-chave de avaliação mais importante na dimensão Capital humano, assim como, de modo respectivo, Inteligência tecnológica (peso $=0,423$ ) e Processo de apropriação dos ativos de PI (peso $=0,498$ ) destacam-se nas dimensões Contexto estratégico e Recursos e processos.

Apesar da importância dos demais elementos que integram essas dimensões, os dados da Tabela 3 sintetizam a essência da gestão da propriedade intelectual, que, de acordo com a literatura, os gestores, pautados nas diretrizes organizacionais e nas informações do mercado, devem criar um ambiente organizacional focado na proteção e apropriação dos ativos intangíveis, por meio do fortalecimento da cultura de proteção da inovação, a fim de extrair valor dos ativos intelectuais protegidos, a partir da implementação de mecanismos de proteção e apropriação formais e informais (DODGE et al., 2017; FREEL; ROBSON, 2017; LANDRY; AMARA; SAIHI, 2009; MOL; MASUREL, 2011; REITZIG, 2007; THOMÄ; ZIMMERMANN, 2013).

Para cada fator-chave de avaliação apresentados na Tabela 3, foram realizadas comparações paritárias para conjuntos de 4 ou 5 indicadores. Desse modo, na Tabela 4 são apresentadas as matrizes de comparação dos indicadores da dimensão Capital humano.

Tabela 4 - Matrizes de comparação dos indicadores da dimensão Capital humano

\begin{tabular}{|c|c|c|c|c|c|c|c|}
\hline \multirow{4}{*}{ 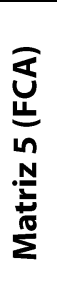 } & Indicadores & QPS & ITD & PRV & EPD & - & Peso \\
\hline & $\begin{array}{l}\text { Qualificação de pessoal para as } \\
\text { ações relativas à PI (QPS) }\end{array}$ & 1,000 & 2,918 & 2,008 & 2,280 & - & 0,439 \\
\hline & $\begin{array}{l}\text { Investimento em atividades de } \\
\text { T\&D (ITD) }\end{array}$ & 0,343 & 1,000 & 1,102 & 1,021 & - & 0,181 \\
\hline & $\begin{array}{l}\text { Percepção da relevância das } \\
\text { ações de PI (PRV) }\end{array}$ & 0,498 & 0,908 & 1,000 & 1,961 & - & 0,223 \\
\hline
\end{tabular}




\begin{tabular}{|c|c|c|c|c|c|c|c|}
\hline & $\begin{array}{l}\text { Engajamento de pessoal em } \\
\text { atividades de P\&D (EPD) }\end{array}$ & 0,439 & 0,979 & 0,510 & 1,000 & - & 0,157 \\
\hline & \multicolumn{7}{|c|}{$\lambda \max =4,065 ;$ I.C. $=0,022 ;$ R.C. $=0,024$} \\
\hline \multirow{7}{*}{ 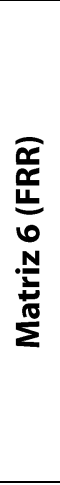 } & Indicadores & PID & INI & RII & RPQ & GAP & Peso \\
\hline & Proposição de novas ideias (PID) & 1,000 & 1,609 & 0,596 & 0,528 & 2,259 & 0,195 \\
\hline & $\begin{array}{l}\text { Implementação de novas ideias } \\
\text { (INI) }\end{array}$ & 0,621 & 1,000 & 1,158 & 0,581 & 0,826 & 0,153 \\
\hline & $\begin{array}{l}\text { Recompensa por ideias } \\
\text { inovadoras (RII) }\end{array}$ & 1,678 & 0,863 & 1,000 & 0,505 & 1,227 & 0,185 \\
\hline & $\begin{array}{l}\text { Retenção de pessoal qualificado } \\
\text { (RPQ) }\end{array}$ & 1,895 & 1,720 & 1,980 & 1,000 & 2,649 & 0,334 \\
\hline & $\begin{array}{l}\text { Grau de atratividade de pessoal } \\
\text { (GAP) }\end{array}$ & 0,443 & 1,210 & 0,815 & 0,378 & 1,000 & 0,132 \\
\hline & \multicolumn{7}{|c|}{$\lambda \max =5,161 ;$ I.C. $=0,040 ;$ R.C. $=0,036$} \\
\hline \multirow{6}{*}{ 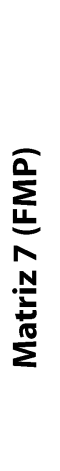 } & Indicadores & MCP & SAP & MDP & UID & $\mathrm{PCl}$ & Peso \\
\hline & $\begin{array}{l}\text { Mapeamento de competências } \\
(\mathrm{MCP})\end{array}$ & 1,000 & 2,338 & 1,590 & 1,139 & 3,071 & 0,309 \\
\hline & $\begin{array}{l}\text { Sistematização da atuação do } \\
\text { pessoal (SAP) }\end{array}$ & 0,428 & 1,000 & 0,721 & 0,755 & 0,643 & 0,127 \\
\hline & $\begin{array}{l}\text { Mensuração do desempenho de } \\
\text { pessoal (MDP) }\end{array}$ & 0,629 & 1,387 & 1,000 & 0,454 & 3,052 & 0,192 \\
\hline & $\begin{array}{l}\text { Uso das informações do } \\
\text { desempenho (UID) }\end{array}$ & 0,878 & 1,324 & 2,202 & 1,000 & 2,166 & 0,261 \\
\hline & $\begin{array}{l}\text { Protocolo de controle da } \\
\text { informação (PCI) }\end{array}$ & 0,326 & 1,556 & 0,328 & 0,462 & 1,000 & 0,111 \\
\hline
\end{tabular}

$\lambda \max =5,190 ; \mathbf{I} \cdot \mathbf{C} .=0,047 ; \boldsymbol{R} . \mathbf{C} .=0,042$

Fonte: Elaborado pelos autores (2019).

De acordo com os dados apresentados nesta Tabela 4, o indicador Qualificação de pessoal para as ações relativas à $\mathrm{PI}(\mathrm{Peso}=0,439)$ tem maior relevância no âmbito do fator-chave Cultura e aprendizagem. Da mesma forma que os indicadores Retenção de pessoal qualificado (Peso $=0,334$ ) e Mapeamento de competências (Peso $=0,309$ ) destacam-se, respectivamente, nos fatores-chave Recompensa e retenção e Monitoramento de pessoal. Esses dados estão de acordo com o posicionamento de Thomä e Zimmermann (2013). Para esses autores, manter as pessoas qualificadas na empresa é um importante mecanismo informal de proteção e apropriação da inovação. Agostini, Nosella e Soranzo (2015) corroboram a opinião de Thomä e Zimmermann (2013). Segundo elas, inovações desenvolvidas com base nas competências únicas das pessoas e lançadas rapidamente no mercado geram maiores lucros para as PMEs.

As comparações paritárias dos indicadores que integram os fatores-chave de avaliação da dimensão Contexto estratégico são apresentas na Tabela 5.

Tabela 5 - Matrizes de comparação dos indicadores da dimensão Contexto estratégico

\begin{tabular}{|c|c|c|c|c|c|c|c|}
\hline \multirow{6}{*}{ 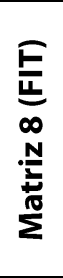 } & Indicadores & ARO & PRT & MTC & ANI & - & Peso \\
\hline & Avaliação de riscos e oportunidades (ARO) & 1,000 & 1,327 & 0,961 & 3,308 & - & 0,334 \\
\hline & Prospecção tecnológica (PRT) & 0,753 & 1,000 & 0,808 & 3,287 & - & 0,277 \\
\hline & Monitoramento da concorrência (MTC) & 1,040 & 1,237 & 1,000 & 1,475 & - & 0,273 \\
\hline & Aplicabilidade das novas ideias (ANI) & 0,302 & 0,304 & 0,678 & 1,000 & - & 0,116 \\
\hline & \multicolumn{7}{|c|}{$\lambda \max =4,101 ; \mathbf{I} . \mathbf{C} .=0,034 ;$ R.C. $=0,037$} \\
\hline \multirow{6}{*}{ 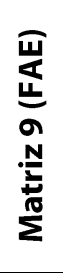 } & Indicadores & RLE & RLF & COE & PRU & RLC & Peso \\
\hline & Relacionamento com especialistas (RLE) & 1,000 & 2,061 & 0,560 & 0,386 & 1,434 & 0,165 \\
\hline & Relacionamento com fornecedores (RLF) & 0,485 & 1,000 & 0,614 & 0,343 & 0,776 & 0,109 \\
\hline & Cooperação com empresas (COE) & 1,786 & 1,628 & 1,000 & 0,669 & 1,517 & 0,224 \\
\hline & Parcerias com universidades (PRU) & 2,593 & 2,915 & 1,496 & 1,000 & 3,347 & 0,374 \\
\hline & Relacionamento com clientes (RLC) & 0,698 & 1,289 & 0,659 & 0,299 & 1,000 & 0,128 \\
\hline
\end{tabular}




\begin{tabular}{|c|c|c|c|c|c|c|c|}
\hline \multirow{6}{*}{ 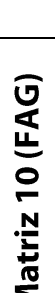 } & \multicolumn{7}{|c|}{$\lambda \max =5,063 ;$ I.C. $=0,016 ;$ R.C. $=0,014$} \\
\hline & Indicadores & AST & ALT & ALI & AES & - & Peso \\
\hline & Alinhamento estratégico (AST) & 1,000 & 1,399 & 2,767 & 2,691 & - & 0,399 \\
\hline & Alinhamento tático (ALT) & 0,715 & 1,000 & 2,381 & 2,721 & - & 0,326 \\
\hline & Alinhamento informacional (ALI) & 0,361 & 0,420 & 1,000 & 0,707 & - & 0,127 \\
\hline & Alinhamento estrutural (AES) & 0,372 & 0,368 & 1,414 & 1,000 & - & 0,147 \\
\hline
\end{tabular}

$\lambda \max =4,028 ;$ I.C. $=0,009 ; \boldsymbol{R} . \boldsymbol{C} .=0,010$

Fonte: Elaborado pelos autores (2019).

Os dados mostrados na Tabela 5 apontam que Avaliação de riscos e oportunidades (Peso =0,334), Parcerias com universidades (Peso $=0,374$ ) e Alinhamento estratégico (Peso =0,399) são os indicadores mais importantes dos fatores-chave de avaliação Inteligência tecnológica, Articulação externa e Alinhamento gerencial, respectivamente. $O$ destaque para esses três indicadores na dimensão Contexto estratégico reforçam os achados da literatura. Para Tietze, Granstrand e Herstatt (2006), por exemplo, a gestão da propriedade intelectual alinhada à estratégia organizacional é o principal desafio na gestão das empresas que, além de lidar com os bens de PI desenvolvidos internamente, deve se preocupar com a aquisição e exploração de tecnologia externa, incluindo um conjunto extenso de tarefas (avaliação de riscos e oportunidades do mercado, celebração de parcerias com instituições de ensino e pesquisa, cooperação com empresas etc.).

Por fim, na Tabela 6 são apresentadas as matrizes de comparação paritária dos indicadores que integram a dimensão Recursos e processos.

Tabela 6 - Matrizes de comparação dos indicadores da dimensão Recursos e processos

\begin{tabular}{|c|c|c|c|c|c|c|c|}
\hline \multirow{7}{*}{ 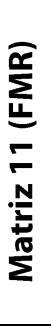 } & Indicadores & IEO & IPD & IAP & IAC & ISG & Peso \\
\hline & Infraestrutura organizacional (IEO) & 1,000 & 1,331 & 0,425 & 0,925 & 1,045 & 0,159 \\
\hline & Investimento em atividades de P\&D (IPD) & 0,751 & 1,000 & 0,407 & 0,983 & 2,943 & 0,175 \\
\hline & Investimento em atividades de proteção de PI (IAP) & 2,354 & 2,457 & 1,000 & 1,525 & 4,057 & 0,367 \\
\hline & Investimento para aquisição de conhecimento (IAC) & 1,081 & 1,017 & 0,656 & 1,000 & 3,308 & 0,214 \\
\hline & Investimento em softwares de gestão (ISG) & 0,957 & 0,340 & 0,246 & 0,302 & 1,000 & 0,085 \\
\hline & \multicolumn{7}{|c|}{$\lambda \max =5,121 ;$ I.C. $=0,030 ;$ R.C. $=0,027$} \\
\hline \multirow{7}{*}{$\begin{array}{l}\frac{a}{0} \\
\frac{1}{4} \\
\frac{N}{2} \\
\frac{N}{2} \\
\frac{\pi}{2}\end{array}$} & Indicadores & OPP & EPP & MPP & GPA & ADP & Peso \\
\hline & Oportunidades de proteção de PI (OPP) & 1,000 & 1,458 & 0,525 & 0,587 & 1,059 & 0,165 \\
\hline & Escopo da proteção de PI (EPP) & 0,686 & 1,000 & 0,572 & 0,809 & 1,124 & 0,156 \\
\hline & Mecanismos de proteção de PI (MPP) & 1,906 & 1,749 & 1,000 & 1,982 & 1,939 & 0,319 \\
\hline & Gestão do portfólio de ativos PI (GPA) & 1,704 & 1,236 & 0,505 & 1,000 & 1,688 & 0,215 \\
\hline & Auditoria da gestão do portfólio de PI (ADP) & 0,945 & 0,889 & 0,516 & 0,593 & 1,000 & 0,146 \\
\hline & \multicolumn{7}{|c|}{$\lambda \max =5,069 ;$ I.C. $=0,017 ;$ R.C. $=0,015$} \\
\hline \multirow{6}{*}{$\begin{array}{l}\frac{a}{\alpha} \\
\frac{1}{4} \\
\frac{m}{n} \\
\frac{N}{2} \\
\frac{\pi}{2}\end{array}$} & Indicadores & PVP & DVE & DEA & OAP & AVP & Peso \\
\hline & Percepção do valor da PI (PVP) & 1,000 & 1,282 & 0,385 & 0,662 & 1,801 & 0,162 \\
\hline & Definição do valor a ser extraído da PI (DVE) & 0,780 & 1,000 & 0,492 & 0,986 & 2,968 & 0,184 \\
\hline & Definição de estratégias de apropriação (DEA) & 2,595 & 2,031 & 1,000 & 1,857 & 4,043 & 0,376 \\
\hline & Organização para a apropriação (OAP) & 1,510 & 1,015 & 0,538 & 1,000 & 1,552 & 0,189 \\
\hline & Avaliação do processo de apropriação (AVP) & 0,555 & 0,337 & 0,247 & 0,644 & 1,000 & 0,089 \\
\hline
\end{tabular}

$\lambda \max =5,074 ;$ I.C. $=0,018 ;$ R.C. $=0,016$

Fonte: Elaborado pelos autores (2019).

De acordo com os dados da Tabela 6, observa-se que, no âmbito do fator-chave Mobilização de recursos, o indicador mais relevante é Investimento em atividades de proteção de $P$ I (Peso =0,367). Do mesmo modo, os indicadores Mecanismos de proteção de $\mathrm{PI}(\mathrm{Peso}=0,319)$ e Definição de estratégias de apropriação (Peso $=0,376)$ foram apontados como mais relevantes no contexto dos fatores-chave Processo de proteção 
dos ativos de Ple Processo de apropriação da Pl, respectivamente. A priorização desses indicadores evidencia um alinhamento com os achados na literatura. Para Eppinger e Vladova (2013), por exemplo, a captura de valor das ações de inovação é fundamental para a competitividade das PMEs, exigindo estratégias deliberadas de proteção e apropriação dos bens de PI. Dessa forma, apesar da limitação de recursos, comum nessas empresas, é importante ter metas de investimentos nas ações de gestão da propriedade intelectual.

Na Tabela 7 é apresentada, para cada indicador que compõe o Índice $E$, uma escala de avaliação valorada por nível. Como mencionado anteriormente, a quantidade de níveis que formam as escalas varia de acordo com o indicador (I). Assim, observam-se indicadores com escala de três, quatro ou cinco níveis. A valoração dos níveis da escala foi realizada com base nos pesos absolutos atribuídos aos indicadores no âmbito do seu respectivo fator-chave (F), conforme os critérios descritos no item 2.2. O peso de cada dimensão (D) foi mantido conforme a comparação paritária apresentada na Tabela 2. A partir destes fez-se a normalização dos pesos dos fatores-chave de avaliação $(F)$, que resultou no fator de ponderação $(w)$, ou seja, $w=F \otimes D$ (Equação 9).

Tabela 7 - Valoração da escala de avaliação dos indicadores que integram o Índice $E$

\begin{tabular}{|c|c|c|c|c|c|c|c|c|c|c|}
\hline \multirow{2}{*}{ Dim. } & \multirow{2}{*}{$\begin{array}{l}\text { Fatores-chave de } \\
\text { avaliação }(F)\end{array}$} & \multirow{2}{*}{$I$} & \multicolumn{5}{|c|}{ Escala de avaliação } & \multicolumn{3}{|c|}{ Pesos } \\
\hline & & & N1 & N2 & N3 & N4 & N5 & $I$ & $w$ & D \\
\hline \multirow{14}{*}{ 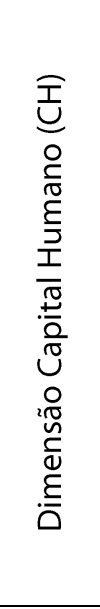 } & \multirow{4}{*}{$\begin{array}{c}\text { Cultura e } \\
\text { aprendizagem (FCA) } \\
(\text { Peso }=0,401)\end{array}$} & QPS & 0,044 & 0,078 & 0,139 & 0,247 & 0,439 & 0,439 & \multirow{4}{*}{0,171} & \multirow{14}{*}{0,428} \\
\hline & & ITD & 0,018 & 0,032 & 0,057 & 0,102 & 0,181 & 0,181 & & \\
\hline & & PRV & 0,022 & 0,040 & 0,070 & 0,125 & 0,223 & 0,223 & & \\
\hline & & EPD & 0,016 & 0,028 & 0,050 & 0,088 & 0,157 & 0,157 & & \\
\hline & \multirow{5}{*}{$\begin{array}{l}\text { Recompensa e } \\
\text { retenção (FRR) } \\
\text { (Peso =0,348) }\end{array}$} & PID & 0,019 & 0,035 & 0,062 & 0,109 & 0,195 & 0,195 & \multirow{5}{*}{0,149} & \\
\hline & & INI & 0,015 & 0,027 & 0,048 & 0,086 & 0,153 & 0,153 & & \\
\hline & & RII & 0,019 & 0,059 & 0,185 & -- & -- & 0,185 & & \\
\hline & & $\mathrm{RPQ}$ & 0,033 & 0,059 & 0,106 & 0,188 & 0,334 & 0,334 & & \\
\hline & & GAP & 0,013 & 0,042 & 0,132 & -- & -- & 0,132 & & \\
\hline & \multirow{5}{*}{$\begin{array}{c}\text { Monitoramento de } \\
\text { pessoal (FMP) } \\
(\text { Peso }=0,252)\end{array}$} & MCP & 0,031 & 0,098 & 0,309 & -- & -- & 0,309 & \multirow{5}{*}{0,108} & \\
\hline & & SAP & 0,013 & 0,040 & 0,127 & -- & -- & 0,127 & & \\
\hline & & MDP & 0,019 & 0,061 & 0,192 & -- & -- & 0,192 & & \\
\hline & & UID & 0,026 & 0,082 & 0,261 & -- & -- & 0,261 & & \\
\hline & & $\mathrm{PCl}$ & 0,011 & 0,035 & 0,111 & -- & -- & 0,111 & & \\
\hline \multirow{13}{*}{ 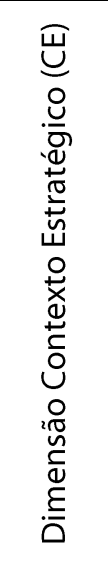 } & \multirow{4}{*}{$\begin{array}{c}\text { Inteligência } \\
\text { tecnológica (FIT) } \\
(\text { Peso }=0,423)\end{array}$} & ARO & 0,033 & 0,105 & 0,334 & -- & -- & 0,334 & \multirow{4}{*}{0,128} & \multirow{13}{*}{0,303} \\
\hline & & PRT & 0,028 & 0,088 & 0,277 & -- & -- & 0,277 & & \\
\hline & & MTC & 0,027 & 0,086 & 0,273 & -- & -- & 0,273 & & \\
\hline & & ANI & 0,012 & 0,037 & 0,116 & -- & -- & 0,116 & & \\
\hline & \multirow{5}{*}{$\begin{array}{c}\text { Articulação Externa } \\
\text { (FAE) } \\
(\text { Peso }=0,332,)\end{array}$} & RLE & 0,017 & 0,052 & 0,165 & -- & -- & 0,165 & \multirow{5}{*}{0,101} & \\
\hline & & RLF & 0,011 & 0,034 & 0,109 & -- & -- & 0,109 & & \\
\hline & & $\mathrm{COE}$ & 0,022 & 0,071 & 0,224 & -- & -- & 0,224 & & \\
\hline & & PRU & 0,037 & 0,118 & 0,374 & -- & -- & 0,374 & & \\
\hline & & $\mathrm{RLC}$ & 0,013 & 0,028 & 0,059 & 0,128 & -- & 0,128 & & \\
\hline & \multirow{4}{*}{$\begin{array}{c}\text { Alinhamento } \\
\text { gerencial (FAG) } \\
(\text { Peso }=0,245)\end{array}$} & AST & 0,040 & 0,126 & 0,399 & -- & -- & 0,399 & \multirow{4}{*}{0,074} & \\
\hline & & ALT & 0,033 & 0,103 & 0,326 & -- & -- & 0,326 & & \\
\hline & & ALI & 0,013 & 0,040 & 0,127 & -- & -- & 0,127 & & \\
\hline & & AES & 0,015 & 0,047 & 0,147 & -- & -- & 0,147 & & \\
\hline \multirow{10}{*}{ 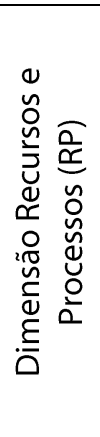 } & \multirow{5}{*}{$\begin{array}{l}\text { Mobilização de } \\
\text { recursos (FMR) } \\
\text { (Peso }=0,217)\end{array}$} & IEO & 0,016 & 0,050 & 0,159 & -- & -- & 0,159 & \multirow{5}{*}{0,058} & \\
\hline & & IPD & 0,018 & 0,031 & 0,055 & 0,098 & 0,175 & 0,175 & & \\
\hline & & IAP & 0,037 & 0,065 & 0,116 & 0,206 & 0,367 & 0,367 & & \\
\hline & & IAC & 0,021 & 0,038 & 0,068 & 0,120 & 0,214 & 0,214 & & \\
\hline & & ISG & 0,009 & 0,027 & 0,085 & -- & -- & 0,085 & & 0,260 \\
\hline & & OPP & 0,016 & 0,052 & 0,165 & -- & -- & 0,165 & & $0,<03$ \\
\hline & Processo de proteção & EPP & 0,016 & 0,049 & 0,156 & -- & -- & 0,156 & & \\
\hline & dos ativos de PI (FPP) & MPP & 0,032 & 0,101 & 0,319 & -- & -- & 0,319 & 0,077 & \\
\hline & $($ Peso $=0,285)$ & GPA & 0,022 & 0,068 & 0,215 & -- & -- & 0,215 & & \\
\hline & & ADP & 0,015 & 0,046 & 0,146 & -- & -- & 0,146 & & \\
\hline
\end{tabular}




\begin{tabular}{|c|c|c|c|c|c|c|c|c|}
\hline \multirow{5}{*}{$\begin{array}{c}\text { Processo de } \\
\text { apropriação da PI } \\
\text { (FPA) } \\
(\text { Peso }=0,498)\end{array}$} & PVP & 0,016 & 0,051 & 0,162 & -- & -- & 0,162 & \multirow{5}{*}{0,134} \\
\hline & DVE & 0,018 & 0,058 & 0,184 & -- & -- & 0,184 & \\
\hline & DEA & 0,038 & 0,119 & 0,376 & -- & -- & 0,376 & \\
\hline & OAP & 0,019 & 0,060 & 0,189 & -- & -- & 0,189 & \\
\hline & AVP & 0,009 & 0,019 & 0,041 & 0,089 & -- & 0,089 & \\
\hline
\end{tabular}

Fonte: Elaborado pelos autores (2019).

O Índice $E$ representa uma função do desempenho das PMEs nos nove fatores-chave de avaliação descritos no Quadro 2, conforme Equação 10:

$$
\text { Índice } E=f\left(F_{1}, F_{2}, F_{3}, F_{4}, F_{5}, F_{6}, F_{7}, F_{8}, F_{9}\right)
$$

Considerando-se que a composição do Indice $E$ assume características aditivas, tem-se sua representação nas Equações 11 e 12, em que $k$ representa o posicionamento de uma PME em um dos níveis da escala de avaliação de cada indicador ( $\boldsymbol{I}$. Dessa forma, cada fator-chave de avaliação $(\boldsymbol{F})$ gera uma medidasíntese intermediária (Índice $F_{i}$ ), resultante do somatório dos desempenhos nos indicadores que o integram.

$$
\begin{gathered}
\text { Indice } F_{i}=k_{1}+k_{2}+\cdots+k_{n}\left(0,1 \leq F_{i} \leq 1\right) \text {, para } \mathrm{n}=4 \text { ou } \mathrm{n}=5 \\
\text { Índice } E=F_{1} w_{1}+F_{2} w_{2}+F_{3} w_{3}+F_{4} w_{4}+F_{5} w_{5}+F_{6} w_{6}+F_{7} w_{7}+F_{8} w_{8}+F_{9} w_{9},
\end{gathered}
$$

sendo cada fator-chave identificado pela notação $i(i=1, \ldots, 9)$, como pode ser visto na forma agregada na Equação 13:

$$
\text { Índice }=\sum_{i=1}^{9} F_{i} W_{i}
$$

A inclusão do fator de ponderação $w$ foi necessária para nivelar o Índice $E$ (que resultaria em no máximo 0,9 , caso fosse utilizada apenas um somatório simples) ao valor das medidas-síntese intermediárias (Índice $F_{i}$ ), que varia de 0,1 a 1,0.

Para classificar o nível de eficiência das PMEs na gestão da propriedade intelectual, propõe-se associar os índices ( $F_{i}$ e E) a graus pré-definidos. Esses graus foram estabelecidos no intervalo de 0,1 a 1,0 (valores que representam o mais baixo e o mais alto desempenho possível para os índices). Os valores dos limites superiores intermediários foram calculados pela soma do valor do limite inferior com uma constante $r(r=0,180)$, resultante da divisão da amplitude total $(1,0 \ominus 0,1)$ pela quantidade de graus pré-definidos (5). Dessa forma, a Tabela 8 apresenta cinco graus de classificação (I, II, III, IV e V), com seus respectivos limites de referência e conceito de desempenho.

Tabela 8 - Graus para classificação da eficiência das PMEs na gestão PI

\begin{tabular}{c|c|c}
\hline Graus & Limites & Conceitos \\
\hline I & $0,100 \leq$ Índice $F_{i}$ ou $E \leq 0,280$ & Desempenho insuficiente \\
\hline II & $0,280<$ Índice $F_{i}$ ou $E \leq 0,460$ & Desempenho básico \\
\hline III & $0,460<$ Índice $F_{i}$ ou $E \leq 0,640$ & Desempenho mediano \\
\hline IV & $0,640<$ Índice $F_{i}$ ou $E \leq 0,820$ & Desempenho bom \\
\hline V & $0,820<$ Índice $F_{i}$ ou $E \leq 1,000$ & Desempenho excelente \\
\hline
\end{tabular}

Fonte: Elaborado pelos autores (2019). 
O enquadramento do Índice $F_{i}$ (relativo a um dos nove fatores-chave de avaliação) e do Índice $E$ (relativo ao desempenho da empresa na totalidade dos fatores-chave de avaliação) nos graus da Tabela 8 permite a identificação de quais aspectos relacionados à gestão da propriedade intelectual merecem maior atenção por parte da empresa avaliada, visto que se pode comparar os índices dos fatores-chave entre si e estes com o índice geral. Permite ainda uma análise do desempenho da empresa ao longo tempo, a partir da verificação da série histórica dos referidos índices.

\section{CONSIDERAÇÕES FINAIS}

As PMEs, normalmente, não possuem ferramentas gerenciais para analisar, de forma adequada, as variáveis relativas à gestão da propriedade intelectual, que impactam na competitividade (LIMA; SANTOS, 2018). Por essa razão e considerando que a avaliação do desempenho organizacional é uma atividade essencial para o suporte no processo decisório, neste trabalho foi aplicado o método Analytic Hierarchy Process (AHP) para agregar, em uma medida-síntese (intitulada Índice E), um conjunto de indicadores. Para tanto, por meio do referido método, atribuíram-se pesos aos elementos ( 3 dimensões, 9 fatores-chaves de avaliação e 42 indicadores) de um modelo teórico previamente validado.

A comparação paritária, realizada para atribuir pesos aos elementos, foi conduzida de acordo com a importância relativa de cada um desses elementos em seu respectivo nível, criando-se, dessa forma, matrizes recíprocas de decisão. No total, foram formadas 13 matrizes de julgamento (uma para as dimensões, que foram comparadas entre si; três para os fatores-chave de avaliação, que foram agrupados por dimensão; e nove para a comparação dos indicadores, agrupados por fator-chave de avaliação). Após a atribuição de pesos, realizouse a valoração da escala de avaliação de cada indicador no âmbito do seu respectivo fator-chave.

Instrumentos gerenciais desenvolvidos para avaliar o desempenho da gestão da propriedade intelectual no âmbito das PMEs são incipientes. Os dois trabalhos identificados na literatura - Enjolras et al., (2014) e Petit et al., (2011) - utilizam escalas lineares simples para mensurar as empresas avaliadas. Diferente desses trabalhos, na presente proposta a valoração da escala de cada indicador foi definida com base na ponderação dos pesos atribuídos a eles pelo método AHP, garantindo maior robustez ao índice.

A principal contribuição deste estudo é a proposição de uma ferramenta que agrega um arcabouço expressivo de indicadores gerenciais, tendo como foco a melhoria do desempenho das PMEs, a partir da identificação de suas prioridades e limitações, fornecendo uma diretriz para a otimização dos seus processos internos, no tocante a gestão da propriedade intelectual. A abrangência das informações que podem ser geradas por essa ferramenta, com base na comparação do nível de eficiência da empresa na gestão da PI em nove fatores-chave de avaliação, pode ser considerada, portanto, um dos pontos fortes do índice proposto.

Os procedimentos metodológicos usados neste estudo podem ser aplicados em uma série de situações, especialmente como suporte na elaboração de indicadores e na valoração de escalas de avaliação de desempenho, já que combina métodos científicos amplamente utilizados no meio acadêmico e corporativo. Apesar da consistência dos procedimentos metodológicos adotados, uma limitação da presente pesquisa está no fato de que o índice proposto não foi testado em nenhuma empresa, de forma que as contribuições do estudo são apenas teóricas.

Para o futuro, espera-se que pesquisas sejam realizadas com foco na aplicação do instrumento aqui proposto em diferentes grupos de PMEs, a fim de gerar uma análise estatística resultante da comparação do diagnóstico de diferentes perfis de empresas (localização, área de atuação etc.). Além de contribuir com a literatura por meio de vários estudos de casos de referência, bem como para o aperfeiçoamento da presente ferramenta de diagnóstico, os resultados agregados dessas pesquisas poderão ser usados por empresas com perfis semelhantes, para a melhoria no processo de tomada de decisão; por órgãos de fomento, para análise de indicadores; e pelo governo, para desenvolver políticas nacionais e regionais com vistas a otimização das ações de inovação em PMEs. 


\section{REFERÊNCIAS}

ACKERMAN, P. Intellectual Property Audit and Management. In: PARR, Russel L.; SMITH, Gordon V. Intellectual Property: Valuation, Exploitation, and Infringement Damages. 5. ed. Hoboken, NJ, USA: John Wiley \& Sons, Inc., 2018. p. 352-371.

AGOSTINI, L.; NOSELLA, A. A dual knowledge perspective on the determinants of SME patenting: results of an empirical investigation. Management Decision, v. 55, n. 6, p. 1226-1247, 2017.

AGOSTINI, L.; NOSELLA, A.; SORANZO, B. The impact of formal and informal appropriability regimes on SME profitability in medium high-tech industries. Technology Analysis \& Strategic Management, v. 27, n. 4, p. 405-419, 21 abr. 2015.

BELINGHERI, P.; LEONE, M. I. Walking into the room with IP: exploring start-ups' IP licensing strategy. Management Decision, v. 55, n. 6, p. 1209-1225, 10 jul. 2017.

BELLEN, H. M. VAN. Indicadores de sustentabilidade: uma análise comparativa. Rio de Janeiro: FGV, 2005. BOGETOFT, P. Performance benchmarking: Measuring and managing performance. New York: Springer, 2013.

BROOKING, A. On the Importance of Managing Intangible Assets as Part of Corporate Strategy. Electronic Journal of Knowledge Management. v. 8, n. 2, p. 217-224, 2010

CANDELIN-PALMQVIST, H.; SANDBERG, B.; MYLLY, U. Intellectual property rights in innovation management research: A review. Technovation, v. 32, n. 9-10, p. 502-512, 2012.

CHEN, T. Expert Systems with Applications Value ontology-based multi-aspect intellectual asset valuation method for decision-making support in k-commerce. Expert Systems With Applications, v. 38, n. 5, p. 5471$5485,2011$.

CLARKE, J.; TURNER, P. Extending the Knowledge-based View: An Examination of Intellectual Property Strategies in Australian Biotechnology Firms. Prometheus, v. 21, n. 1, p. 85-100, 18 jan. 2003.

COHEN, J. R. et al. Corporate Reporting of Nonfinancial Leading Indicators of Economic Performance and Sustainability. Accounting Horizons, v. 26, n. 1, p. 65-90, mar. 2012.

DELERUE, H.; LEJEUNE, A. Managerial secrecy and intellectual asset protection in SMEs: The role of institutional environment. Journal of International Management, v. 17, n. 2, p. 130-142, jun. 2011.

DEV, N. K. et al. Multi-criteria evaluation of real-time key performance indicators of supply chain with consideration of big data architecture. Computers and Industrial Engineering, v. 128, p. 1076-1087, Apr. 2019.

DODGE, R. et al. The Role of Leadership in Innovation: A quantitative analysis of a large data set examines the relationship between organizational culture, leadership behaviors, and innovativeness. ResearchTechnology Management, v. 60, n. 3, p. 22-29, 2017.

EJDYS, J. Future Oriented Strategy for SMEs. Procedia - Social and Behavioral Sciences, v. 156, p. 8-12, Apr. 2014.

ENJOLRAS, M. et al. Proposal of a methodology to elicit maturity curves: Application to innovation and protection capabilities of SMEs. In: INTERNATIONAL CONFERENCE ON ENGINEERING, TECHNOLOGY AND INNOVATION (ICE), Bergamo, 2014. Proceedings [...]. Bergamo, Italy: IEEE, 2014.

EPPINGER, E.; VLADOVA, G. Intellectual property management practices at small and medium-sized enterprises. International Journal of Technology Management, v. 61, n. 1, p. 64-81, 2013.

FORMAN, E.; PENIWATI, K. Aggregating individual judgments and priorities with the Analytic Hierarchy Process. European Journal of Operational Research, v. 108, n. 1, p. 165-169, 1998.

FREEL, M.; ROBSON, P. J. Appropriation strategies and open innovation in SMEs. International Small Business Journal: Researching Entrepreneurship, v. 35, n. 5, p. 578-596, 2017. 
HALL, B. H.; SENA, V. Appropriability mechanisms, innovation, and productivity: evidence from the UK. Economics of Innovation and New Technology, v. 26, n. 1-2, p. 42-62, 17 fev. 2017.

HARRISON, S. S.; SULLIVAN, P. H. Edison in the Boardroom Revisited: How Leading Companies Realize Value from Their Intellectual Property. 2. ed. [s.l.] John Wiley \& Sons, 2011.

HOLGERSSON, M. Patent management in entrepreneurial SMEs: a literature review and an empirical study of innovation appropriation, patent propensity, and motives. R\&D Management, v. 43, n. 1, p. 21-36, jan. 2013.

HU, M.; TSAI, C. Building External Network on Intellectual Property Through Joint R\&D Service Outsourcing: The Case of Taiwan's SMEs. In: TECHNOLOGY MANAGEMENT FOR THE GLOBAL FUTURE (PICMET), Istambul, 2006. Proceedings [...]. Istambul, Turkey: IEEE, 2006.

HUANG, H.-C.; LAI, M.-C.; LIN, L.-H. Developing strategic measurement and improvement for the biopharmaceutical firm: Using the BSC hierarchy. Expert Systems with Applications, v. 38, n. 5, p. 48754881, maio 2011.

JANNUZZI, P. DE M. Indicadores para diagnóstico, monitoramento e avaliação de programas sociais no Brasil. Revista do Serviço Público, v. 56, n. 2, p. 137-160, 2005.

JOUNG, C. B. et al. Categorization of indicators for sustainable manufacturing. Ecological Indicators, v. 24, p. 148-157, jan. 2013.

KAPLAN, R. S.; NORTON, D. P. Balanced Scorecard. In: BOERSCH, Cornelius; ESCHEN, Rainer. Das Summa Summarum des Management. Wiesbaden: Gabler, 2007. p. 137-148.

KELLI, A. et al. Trade Secrets in the Intellectual Property Strategies of Entrepreneurs: The Estonian Experience. Review of Central and East European Law, v. 35, n. 4, p. 315-339, 1 nov. 2010.

KITCHING, J.; BLACKBURN, R. Intellectual property management in the small and medium enterprise (SME). Journal of Small Business and Enterprise Development, v. 5, n. 4, p. 327-335, dez. 1998.

KÖHLER, F. Patent cross-licensing, the influence of IP interdependency and the moderating effect of firm size. The Journal of Technology Transfer, v. 36, n. 4, p. 448-467, 4 ago. 2011.

LANDRY, R.; AMARA, N.; SAIHI, M. Complementarities between strategies to protect inventions and innovations: evidence from manufacturing SMEs. International Journal of Intellectual Property Management, v. 3, n. 1, p. 56-78, 2009.

LAWSON, B.; SAMSON, D.; RODEN, S. Appropriating the value from innovation: inimitability and the effectiveness of isolating mechanisms. Inimitability and the effectiveness of isolating mechanisms, v. 42, n. 5, p. 420-434, 2012.

LEE, Y. et al. Technology opportunity identification customized to the technological capability of SMEs through two-stage patent analysis. Scientometrics, v. 100, n. 1, p. 227-244, 22 jul. 2014.

LEIPONEN, A.; BYMA, J. If you cannot block, you better run: Small firms, cooperative innovation, and appropriation strategies. Research Policy, v. 38, n. 9, p. 1478-1488, nov. 2009.

LIMA, F. V. R. Índice de eficiência da gestão da propriedade intelectual em pequenas e médias empresas: 2020. 147 f.Tese (Doutorado em Ciência da Propriedade Intelectual) - Universidade Federal de Sergipe, São Cristóvão, SE, 2019.

LIMA, F. V. R.; SANTOS, J. A. B. Intellectual Property Management in Small and Medium-Sized Enterprises: A Systematic Literature Review. International Journal for Innovation Education and Research, v. 6, n. 09, p. 109-127, 2018.

LITTMANN-HILMER, G.; KUCKARTZ, M. SME tailor-designed patent portfolio analysis. World Patent Information, v. 31, n. 4, p. 273-277, 2009.

LO-IACONO-FERREIRA, V. G.; CAPUZ-RIZO, S. F.; TORREGROSA-LÓPEZ, J. I. Key Performance Indicators to optimize the environmental performance of Higher Education Institutions with environmental management system - A case study of Universitat Politècnica de València. Journal of Cleaner Production, v. 178, p. 846865, 2018. 
$M A$, Z. et al. Constructing road safety performance indicators using fuzzy delphi method and grey delphi method. Expert Systems with Applications, v. 38, n. 3, p. 1509-1514, 2011.

MAHMOOD, K. et al. A Performance Evaluation Concept for Production Systems in an SME Network. Procedia CIRP, v. 72, p. 603-608, 2018.

MALDONADO-GUZMÁN, G. et al. Knowledge management as intellectual property: Evidence from Mexican Manufacturing SMEs. Management Research Review, v. 39, n. 7, p. 830-850, 18 jul. 2016.

MARDANI, A. et al. A Combined Hybrid Fuzzy Multiple Criteria Decision-making Approach to Evaluating of QM Critical Success Factors in SME's Hotels Firms. Procedia - Social and Behavioral Sciences, v. 172, p. 786793, 2015.

MARRAS, J. P. Administração de remuneração. 2. ed. São Paulo: Pearson Education do Brasil, 2012.

MOL, M. C.; MASUREL, E. Innovation protection by SMEs: the case of the north east of the Netherlands. International Journal of Intellectual Property Management, v. 4, n. 3, p. 153-164, 2011.

NEELY, A. The evolution of performance measurement research. International Journal of Operations \& Production Management, v. 25, n. 12, p. 1264-1277, dez. 2005.

OCDE - ORGANIZAÇÃO PARA A COOPERAÇÃO E DESENVOLVIMENTO ECONÔMICO. Environmental Indicators - Development, Measurement and Use. Paris: OCDE, 2003.

PARK, S. Analyzing the efficiency of small and medium-sized enterprises of a national technology innovation research and development program. SpringerPlus, v. 3, n. 1, p. 403, 4 dez. 2014.

PETIT, C. et al. A new, innovative and marketable IP diagnosis to evaluate, qualify and find insights for the development of SMEs IP practices and use, based on the AIDA approach. World Patent Information, v. 33, n. 1, p. 42-50, 2011.

PFISTER, J. A.; JACK, S. L.; DARWIN, S. N. Strategizing open innovation: How middle managers work with performance indicators. Scandinavian Journal of Management, v. 33, n. 3, p. 139-150, 2017.

REGAZZONI, D.; RIZZI, C.; NANI, R. A TRIZ-based approach to manage innovation and intellectual property. International Journal of Technology Management, v. 55, n. 3/4, p. 274-285, 2011.

REHMAN, M. H. U. et al. Big data reduction framework for value creation in sustainable enterprises. International Journal of Information Management, v. 36, n. 6, p. 917-928, 2016.

REITZIG, M. How executives can enhance IP strategy and performance. MIT Sloan management review, $v$. 49, n. 1, p. 37-43, 2007.

SAATY, T. L. How to make a decision: The analytic hierarchy process. European Journal of Operational Research, v. 48, n. 1, p. 9-26, 1990.

SAUNILA, M. Performance measurement approach for innovation capability in SMEs. International Journal of Productivity and Performance Management, v. 65, n. 2, p. 162-176, 8 fev. 2016.

SCHAUTEN, M.; STEGINK, R. The discount rate for discounted cash flow valuations of intangible assets. Managerial Finance, v. 36, n. 9, p. 799-811, 2013.

SEKHAR, C.; PATWARDHAN, M.; VYAS, V. A Delphi-AHP-TOPSIS Based Framework for the Prioritization of Intellectual Capital Indicators: A SMEs Perspective. Procedia - Social and Behavioral Sciences, v. 189, p. 275-284, 2015.

SEO, H. et al. Value capture mechanism: R\&D productivity comparison of SMEs. Management Decision, v. 53, n. 2, p. 318-337, 2015.

SEO, H. G. et al. SME's appropriability regime for sustainable development-the role of absorptive capacity and inventive capacity. Sustainability , Switzerland, v. 8, n. 7, p. 1-16, 2016.

SHANG, C.; QIU, X.; WEN, Q. Research on the evaluation of intellectual property financing capacity of technology-based SMEs. Boletin Tecnico/Technical Bulletin, v. 55, n. 20, p. 481-488, 2017. 
SHI, Z. et al. Smes' Performance Evaluation and Optimization Based on DEA and CFI. Management and Production Engineering Review, v. 4, n. 1, p. 57-64, 1 mar. 2013.

SLACK, N. et al. Gerenciamento de operações e de processos: princípios e prática de impacto estratégico. Porto Alegre: Bookman, 2006.

TALVELA, J. et al. How individual inventors and SMEs exploit intellectual property rights: The case of Finland. In: PORTLAND INTERNATIONAL CONFERENCE ON MANAGEMENT OF ENGINEERING AND TECHNOLOGY (PICMET), Honolulu, 2016. Proceedings [...]. Honolulu, Hawaii, USA: IEEE, 2016. p. 1645-1658.

THOMÄ, J. Zum Aneignungsverhalten innovationsaktiver kleiner und mittlerer Unternehmen.

Wirtschaftsdienst, v. 93, n. 9, p. 629-635, 19 set. 2013.

THOMÄ, J.; ZIMMERMANN, V. Knowledge Protection Practices in Innovating SMEs. Jahrbücher für Nationalökonomie und Statistik, v. 233, n. 5-6, p. 691-717, 1 jan. 2013.

TIDD, J.; BESSANT, J. P. Gestão da Inovação. São Paulo: Bookman Editora, 2015.

TIETZE, F.; GRANSTRAND, O.; HERSTATT, C. Towards Strategic Intellectual Property Management - Events During the Development: Evidence from Biotech SMEs. In: TECHNOLOGY MANAGEMENT FOR THE GLOBAL FUTURE (PICMET), Istambul, 2006. Proceedings [...]. Istambul, Turkey: IEEE, 2006.

VAN RIJNSOEVER, F. J.; KEMPKES, S. N.; CHAPPIN, M. M. H. Seduced into collaboration: A resource-based choice experiment to explain make, buy or ally strategies of SMEs. Technological Forecasting and Social Change, v. 120, p. 284-297, jul. 2017.

WANG, M. K.; HWANG, K. P. Key factors for the successful evaluation and screening of managers of the intellectual property rights speciality. Expert Systems with Applications, v. 38, n. 9, p. 10794-10802, 2011.

WANG, Y.-H. Identifying competitive intelligence of collaborative intellectual property alliances: analytic platform and case studies. Information Systems and e-Business Management, v. 14, n. 3, p. 491-505, 2016.

WEENEN, T. C. et al. PharmaNutrition A decision framework to evaluate intellectual property strategies in the medical nutrition market. Biochemical Pharmacology, v. 1, n. 2, p. 65-72, 2013.

WILLOUGHBY, K. W. Intellectual property management and technological entrepreneurship. International Journal of Innovation and Technology Management, v. 10, n. 06, p. 1340027, dez. 2013.

ZUBIELQUI, G. C. DE; JONES, J.; STATSENKO, L. Managing Innovation Networks for Knowledge Mobility and Appropriability: A Complexity Perspective. Entrepreneurship Research Journal, v. 6, n. 1, p. 75-109, 1 jan. 2016. 